\title{
Efecto de las altas presiones hidrostáticas respecto a la pasteurización térmica en los aspectos microbiológicos, sensoriales y estabilidad oxidativa de un paté de aceituna
}

\author{
Por J. Sánchez ${ }^{\star a}$, C. De Miguel ${ }^{b}$, M. R. Ramírez ${ }^{a}$, J. Delgado $^{a}$, M.N. Franco ${ }^{a}$ y D. Martín ${ }^{a}$ \\ ${ }^{a}$ Instituto Tecnológico Agroalimentario de Extremadura. Avda. Adolfo Suárez s/n. 06071. Badajoz. España. \\ ${ }^{\mathrm{b}}$ UEX. Departamento de Biología Vegetal, Ecología y Ciencias de la Tierra. \\ * Autor para la correspondencia: jacinto.sanchez@juntaextremadura.es
}

\section{RESUMEN}

Efecto de las altas presiones hidrostáticas respecto a la pasteurización térmica en los aspectos microbiológicos, sensoriales y estabilidad oxidativa de un paté de aceituna.

En el presente trabajo se valoró el efecto del procesado por altas presiones hidrostáticas (APH) como método alternativo al tratamiento térmico de pasteurización para la conservación del paté de aceituna y su estabilidad durante el almacenamiento en refrigeración, mediante la caracterización de los aspectos microbiológicos, físico-químicos y sensoriales. El paté de aceituna fue elaborado partiendo de aceituna de mesa verde deshuesada y envasada en salmuera, a la cual, previo lavado con agua, se le adiciona zumo de naranja natural, aceite de oliva virgen, especias y sorbato potásico. Para evaluar el efecto del procesado, se aplicaron cuatro tratamientos de APH (450 y $600 \mathrm{MPa}$ durante 5 y $10 \mathrm{~min})$ y otro de pasteurización térmica $\left(80^{\circ} \mathrm{C}\right.$ durante $\left.20 \mathrm{~min}\right)$, comparándose con el producto no procesado. Las muestras tratadas con APH presentaron, frente a las tratadas por pasteurización térmica, una reducción en la presencia de microorganismos, un aumento de su estabilidad oxidativa, una mayor aceptación sensorial; y respecto a las coordenadas colorimétricas mayor claridad y menor pardeamiento. El estudio de la vida útil del producto en refrigeración, indicaría la viabilidad de la aplicación de la tecnología de APH para obtener alimentos con vida útil similar a la obtenida con el tratamiento tradicional de pasteurización, pero con mejor calidad sensorial.

PALABRAS CLAVE: Altas presiones hidrostáticas (APH) - Análisis sensorial - Estabilidad oxidativa - Microbiológico - Pasteurización térmica - Paté de aceituna.

\section{SUMMARY}

Effect of high hydrostatic pressure versus thermal pasteurization on the microbiological, sensory aspects and oxidative stability of olive pate.

The present study examines the effect of high hydrostatic pressure processing (HHP) as an alternative to thermal pasteurization treatments for the conservation of olive pate and its stability during refrigerated storage, through the characterization of microbiological, physico-chemical and sensory aspects. The olive pate was made with green, pitted table olives packed in brine. Orange juice, olive oil, spices and potassium sorbate were added to the olives after being washed in water. To evaluate the effects of processing, four treatments of APH (450 and 600MPa for 5 and $10 \mathrm{~min}$ ) and a thermal pasteurization $\left(80^{\circ} \mathrm{C}\right.$ for $20 \mathrm{~min}$ ) were applied to compare them with the unprocessed product. APH-treated samples showed a further reduction in the presence of microorganisms, an increase in oxidative stability, a higher sensory acceptance, greater clarity and less browning regarding the colorimetric coordinates, in comparison with those treated by thermal pasteurization. The study of the shelf life of the product in refrigeration would indicate the feasibility to implement APH technology in order to obtain food with a similar shelf life to foods obtained through the traditional thermal pasteurization treatment, but with better sensory quality.

KEY-WORDS: High hydrostatic pressures (HHP) Microbiologic - Olive pate - Oxidative stability - Sensory analysis - Thermal pasteurization.

\section{INTRODUCCIÓN}

En las últimas décadas se han llevado a cabo importantes avances en el conocimiento de tecnologías de conservación y/o transformación de alimentos distintas de las aplicaciones térmicas convencionales. Entre ellas podemos citar los pulsos eléctricos de alta intensidad de campo (SolivaFortuny et al., 2009), la extracción mediante fluidos supercríticos (Reglero et al., 2005), la deshidratación osmótica (Rastogi et al., 2005) o la alta presión hidrostática (Mor-Mur, 2010). La investigación tanto en desarrollo de equipos como en las consecuencias de los nuevos tratamientos ha permitido que algunas de ellas ya estén en el mercado y se empleen industrialmente. En general se las considera como alternativa al procesado térmico con el fin de obtener alimentos seguros que mantengan mejor las características organolépticas o nutricionales.

El procesamiento por alta presión hidrostática $(\mathrm{APH})$ a temperatura de refrigeración, temperatura ambiente o con calentamiento moderado, permite la reducción/inactivación de microorganismos alterantes y patógenos en los alimentos, al tiempo que se evitan las consecuencias negativas de los tratamientos térmicos convencionales (Barbosa-Cánovas et al., 1998; Torres y Velázquez, 2008). La efectividad de este tipo de tratamientos es debida al efecto que las altas presiones tienen sobre la 
morfología, estructura y funcionalidad de las membranas celulares (alteraciones de la permeabilidad celular e intercambio de iones), metabolismo y mecanismos de división de los microorganismos. De este modo, las levaduras, los hongos y las células vegetativas de las bacterias pueden ser inactivados por presiones comprendidas en el rango de los 200 a los $700 \mathrm{MPa}$. En general, las presiones de trabajo oscilan entre 400 y $900 \mathrm{MPa}$. Dicha presión se transmite de forma instantánea y uniforme, independientemente del tamaño, forma o composición del alimento.

Esta tecnología es un método de conservación que se está aplicando en la actualidad a nivel industrial en diferentes productos que se encuentran ya en el mercado, como productos de alto valor nutritivo libres de aditivos: zumos y purés de frutas, guacamole, salsas, tofu, etc. (Porreta et al., 1995; Préstamo y Fontecha, 2008; Voigt et al., 2010). Mediante este tipo de procesado se facilita la conservación de las características organolépticas y sensoriales de los alimentos "frescos" o no procesados, y mantiene el valor nutritivo, por ser un tratamiento menos agresivo para las vitaminas y otros compuestos con actividad antioxidante que juegan un papel primordial para la salud. Esto se debe a que la presión rompe los enlaces por puentes de hidrógeno pero no los enlaces covalentes de los compuestos, lo que evita cambios en la composición química de los alimentos. Esto explica por qué la composición química y el análisis sensorial de los alimentos tratados por altas presiones son similares a los productos no tratados, en cuanto a textura, aroma y color, y los cambios son inferiores a los observados mediante los tratamientos térmicos (Mcinerney et al., 2007; Oey et al., 2007; Oey et al., 2008).

El paté de aceituna es un producto con una elaboración tradicional que se encuadra dentro de los alimentos delicatesen. Por su elaboración con aceitunas, presenta la ventaja de que permite la diversificación del mercado de este producto y contribuye a disminuir los excedentes en el sector. La aplicación del tratamiento térmico para la conservación del paté, en algunos casos, oscurece el producto pudiendo dar lugar a un aspecto poco agradable para los consumidores, sin embargo la aplicación de otro tipo de procesado podría mejorar la calidad final del producto.

El objetivo del presente trabajo es estudiar el efecto que el tipo de tratamiento: APH y térmico tiene sobre la seguridad microbiana, el color, el análisis sensorial y la estabilidad oxidativa en un paté de aceituna; y evaluar la estabilidad del producto durante su almacenamiento a refrigeración.

\section{MATERIALES Y MÉTODOS}

\subsection{Elaboración del paté de aceituna}

El paté fue elaborado partiendo de aceituna de mesa verde deshuesada comercial de la variedad Manzanilla carrasqueña envasada en salmuera a alta concentración. Las condiciones de lavado utilizadas (tiempo y número de cambios de agua) con el fin de obtener aceitunas con un contenido de sal adecuado y atractivo para el consumidor fueron de remojo en agua durante 90 minutos cambiando el agua cada 30 minutos (Escudero-Gilete et al., 2009). A las aceitunas una vez lavadas y escurridas se le adicionó: aceite de oliva virgen de la variedad arbequina $(100 \mathrm{~mL} / \mathrm{kg})$ y mezcla de especias de orégano, tomillo y albahaca (1 cucharada sope$\mathrm{ra} / \mathrm{kg}$ ) para proveer a la pasta de aroma y textura (Escudero-Gilete et al., 2009). Se utilizó como acidificante zumo de naranja natural $(8 \mathrm{~mL} / \mathrm{kg})$ y como conservante sorbato potásico $(50 \mathrm{mg} / \mathrm{kg})$. Todos los ingredientes mencionados se picaron con un robot de cocina (Thermomix TM31-1, Wuppertal, Alemania) a máxima potencia durante 10 minutos. A continuación se realizó el envasado de dos formas diferentes atendiendo a que el tratamiento fuera de Altas Presiones Hidrostáticas (envasado a vacío (Gustav Müller VS 100, Alemania) en bandejas de plástico termoselladas de $250 \mathrm{~g}$ ) o Pasteurización (frascos de vidrio de 125g).

\subsection{Tratamientos Experimentales}

\section{Altas Presiones Hidrostáticas (APH)}

Para la aplicación de los tratamientos se utilizó un equipo comercial de altas presiones hidrostáticas (NC Hyperbaric Wave 6000/55 Burgos, España). Es un equipo industrial con una capacidad de 55L, que aplica un tratamiento máximo de 600MPa/6000bar. La temperatura inicial del agua de tratamiento fue de $10^{\circ} \mathrm{C}$, con un tiempo de compresión menor de 4 minutos. La descompresión de los productos fue prácticamente instantánea.

Los lotes de las bandejas de paté se sometieron a APH aplicándose presiones de 450 y $600 \mathrm{MPa}$ durante un tiempo de tratamiento de 5 y 10 minutos a ambas presiones, suponiendo un total de cuatro tratamientos experimentales.

\section{Tratamientos térmico: Pasteurización}

El paté, envasado en botes de vidrio, fue sometido a tratamiento en autoclave horizontal rotatorio de 300L, fabricado sobre demanda (Chaconsa, Murcia, España) a una temperatura de $40^{\circ} \mathrm{C}$, para posteriormente aumentarse a $80{ }^{\circ} \mathrm{C}$ manteniéndose durante 20 minutos.

\subsection{Análisis}

Se elaboraron cuatro muestras de paté por tratamiento y día de almacenamiento resultando un total de 48 muestras La mitad de las muestras procesadas fueron analizadas al día siguiente del tratamiento térmico o de APH, para evaluar el efecto del tratamiento, mientras que la otra mitad fueron almacenadas en una cámara a refrigeración $\left(4^{\circ} \mathrm{C}\right)$ durante 30 días para evaluar el efecto del almace- 
namiento y de los tratamientos sobre los productos elaborados. Los análisis microbiológicos, sensoriales y parámetros colorimétricos se realizaron en las muestras en fresco; mientras que la estabilidad oxidativa se determinó una vez extraída la grasa con éter etílico y posterior almacenamiento $\mathrm{a}-80^{\circ} \mathrm{C}$.

\section{Análisis microbiológicos}

La cuantificación de los microorganismos aeróbios mesófilos se realizo en medio Agar de Recuento en Placa, PCA (MERCK) incubado durante $72 \mathrm{~h}$ a $30{ }^{\circ} \mathrm{C}$ (ISO 4833:1991). El análisis microbiológico de los microorganismos psicrotrofos viables se realizó en medio Agar de Recuento en Placa PCA, (MERCK) incubado durante 5 días a $16{ }^{\circ} \mathrm{C}$ (Pascual-Anderson y Calderón-Pascual, 2000). Para el recuento de enterobacterias se utilizó el medio Agar Violeta Rojo y Bilis Glucosa, VRBG (MERCK) incubado durante $24 \mathrm{~h}$ a $37^{\circ} \mathrm{C}$ (ISO 7402:1993). La determinación y cuantificación de Escherichia coli se realizó en medio Chromocult (MERCK) incubado durante $24 \mathrm{~h}$ a $37{ }^{\circ} \mathrm{C}$. El recuento de hongos y levaduras se realizó en medio Agar Extracto de Levadura Glucosa Cloranfenicol, YGC (MERCK) incubado durante 5 días a $25^{\circ} \mathrm{C}$ (ISO 7954:1988).

\section{Color instrumental}

El color instrumental se midió en un espectrofotómetro de Konica Minolta CM-3500d (Aquateknica, S.A., España). La medida de la reflectancia se realizó a lo largo de todo el espectro visible (380nm-770nm, $\Delta \lambda=2 \mathrm{~nm}$ ), geometría $\mathrm{d} / 8$, ángulo de visión de $10^{\circ}$, área de medición de $8 \mathrm{~mm}$ (cubetas de cuarzo de $8 \mathrm{~mm}$ de diámetro y $10 \mathrm{~mm}$ de espesor) e iluminación primaria D65. Los parámetros de color correspondientes al espacio CIELAB $\left(L^{*}, a^{*}\right.$ y $\left.b^{*}\right)$ fueron obtenidos directamente del equipo. Además se calcularon los parámetros psicofísicos cromaticidad $\left[\mathrm{C}_{\mathrm{ab}}{ }^{*}=\left(\mathrm{a}^{\star 2}+\mathrm{b}^{\star 2}\right)^{0.5}\right]$ y ángulo del tono o hue $\left[\mathrm{h}_{\mathrm{ab}}{ }^{*}=\operatorname{arc} \tan \left(\mathrm{b}^{*} / \mathrm{a}^{*}\right)(\mathrm{CIE}, 2004)\right.$. Las determinaciones se hicieron por duplicado en cada muestra.

\section{Análisis sensorial}

Los patés elaborados procedentes de los distintos tratamientos experimentales se valoraron organolépticamente por un panel de jueces seleccionados con larga experiencia en el análisis sensorial de alimentos para la investigación y control de calidad (ISO 8586-2:2008). El número de catadores fue de 23 participantes de ambos sexos (equilibrados) y edades comprendidas entre 25 y 55 años. Las evaluaciones se llevaron en las cabinas de pruebas individuales de la sala de catas (ISO 8589:2007). A los catadores se les presentó $20 \mathrm{~g}$ por muestra en bandejas de plástico blancas etiquetadas mediante la codificación de un número de tres dígitos aleatorios. La valoración sensorial se realizó con una ficha elaborada para este estu- dio donde se evalúan los descriptores: aspecto, sabor, textura y valoración global de los patés procesados. Para la valoración de los descriptores los catadores utilizaron una escala no estructurada de $10 \mathrm{~cm}$ de largo donde se aplicó la puntuación para cada paté procesado $(0=$ " no me gusta" hasta 10 = "me gusta mucho"). El estimador de los resultados de la evaluación sensorial fue la mediana. No se cataron las muestras controles.

\section{Estabilidad oxidativa}

A las muestras se le extrajo la grasa con éter etílico y se determinaron los siguientes parámetros: índice de peróxidos determinado siguiendo la metodología descrita en el Reglamento (CEE) 2568/91 de la comisión de 11 de julio de 1991 (DOCE, 1991), y sus posteriores modificaciones, relativo a las características de los aceites de oliva y de los aceites de orujo de oliva y sobre sus métodos de análisis; y estabilidad rancimat usando el equipo Rancimat 679 (Metrohm Co., Basel, Switzerland), aplicando un flujo de aire de $10 \mathrm{~L} / \mathrm{h}$ a $100{ }^{\circ} \mathrm{C}$ de temperatura.

\section{Análisis estadístico}

Para la realización del análisis estadístico se aplicó un análisis de varianza (ANOVA). Cuando las diferencias fueron estadísticamente significativas $(p \leqslant 0.05)$, se utilizó el Test de Tukey. El análisis estadístico de los datos se realizó utilizando el programa estadístico SPSS versión 13.0 de Windows.

\section{RESULTADOS Y DISCUSIÓN}

En la Tabla 1 y 2 se recogen los resultados de inactivación microbiana tras la aplicación del tratamiento térmico y de los tratamientos de alta presión hidrostática en el paté de aceituna el día de elaboración y a los 30 días del mismo, respectivamente. En general, en los patés de aceituna procesados, tanto por APH como por tratamiento térmico, se observa una reducción estadísticamente significativa en los recuentos de microorganismos psicrotrofos con respecto al control sin tratar. La eliminación de los microorganismos fue más intensa en tratamientos a presiones más elevadas (600MPa vs 450MPa).

En cuanto a la inactivación microbiana, y para el día de elaboración, los tratamientos a presiones más elevadas (600MPa vs $450 \mathrm{MPa}$ ) y a mayores tiempos de tratamiento (10min vs $5 \mathrm{~min}$ ) fueron más eficaces. Resultados que corroboran los obtenidos por otros autores (Capellas et al., 1996; Cheftel y Culioli, 1997; Ponce et al., 1998). La aplicación de los diferentes tratamientos (alta presión y térmico), sobre el paté de aceituna fue efectiva para la inactivación de mohos y levaduras, no siendo detectados a día 1 (Tabla 1) en ninguna de las muestras. 
Tabla 1

Cambios microbiológicos (log UFC/gr) en el paté de aceituna tratado con altas presiones hidrostáticas (APH) y mediante tratamiento térmico a día 1

\begin{tabular}{|c|c|c|c|c|c|c|c|c|c|c|c|c|}
\hline \multirow[b]{3}{*}{ Día 1} & \multirow{2}{*}{\multicolumn{2}{|c|}{ Control }} & \multirow{2}{*}{\multicolumn{2}{|c|}{$\begin{array}{l}\text { Tratamiento } \\
\text { térmico }\end{array}$}} & \multicolumn{4}{|c|}{ APH (450 MPa) } & \multicolumn{4}{|c|}{ APH (600 MPa) } \\
\hline & & & & & \multicolumn{2}{|c|}{$5 \mathrm{~min}$} & \multicolumn{2}{|c|}{$10 \mathrm{~min}$} & \multicolumn{2}{|c|}{$5 \mathrm{~min}$} & \multicolumn{2}{|c|}{$10 \mathrm{~min}$} \\
\hline & Media & $D E$ & Media & $D E$ & Media & $D E$ & Media & $D E$ & Media & $D E$ & Media & $D E$ \\
\hline Mesófilos & $5,0 c$ & $\pm 0,0$ & $3,8 b$ & $\pm 0,2$ & $4,0 \mathrm{~b}$ & $\pm 0,1$ & $3,7 b$ & $\pm 0,1$ & $3,6 a b$ & $\pm 0,5$ & $3,1 a$ & $\pm 0,1$ \\
\hline Psicrotrofos & $4,2 c$ & $\pm 0,0$ & $2,5 a$ & $\pm 0,1$ & $3,6 b$ & $\pm 0,4$ & $3,6 b$ & $\pm 0,2$ & $3,6 b$ & $\pm 0,3$ & $2,9 a b$ & $\pm 0,4$ \\
\hline Enterobacterias & nd & & nd & & nd & & nd & & nd & & nd & \\
\hline E. coli & nd & & nd & & nd & & nd & & nd & & nd & \\
\hline Mohos y levaduras & 0,5 & $\pm 0,7$ & nd & & nd & & nd & & nd & & nd & \\
\hline
\end{tabular}

Diferentes letras en la misma fila indican diferencias estadísticamente significativas (Test de Tukey p $<0,05$ ). ns: no significativo. nd: no detectado por debajo del límite de detección. Los resultados se expresan como media y DE (desviación estándar).

Tras el almacenamiento en refrigeración durante un mes, los recuentos microbianos de aerobios mesófilos, psicrotrofos, Escherichia coli y enterobacterias totales se mantuvieron estables en todos los tratamientos aplicados, e incluso en algunos casos se detectó una disminución en el recuento, hecho más destacable en las determinaciones de psicrotrofos de muestras tratadas por APH. Esta disminución se debe a la inducción mediante las altas presiones de daños subletales en algunos microorganismos y que no son capaces de reparar con el tiempo, provocando su muerte (De Lamo et al., 2004). Las altas presiones inducen cambios de tipo morfológico, bioquímico y genético, en la membrana y en la pared celular de los microorganismos, que van a provocar cambios en el funcionamiento y en la reproducción de los microorganismos (Cheftel, 1992; De Lamo et al., 2004), propiciando la muerte de los microorganismos. Asimismo, este menor recuento también puede deberse al pH ácido del producto ( $\mathrm{pH} 3)$, el cual sensibiliza fuertemente a los microorganismos sometidos a altas presiones y favorece que los microorganismos fisiológicamente comprometidos tras el tratamiento de APH no sobrevivan durante el periodo de refrigeración posterior (Carlez, 1994; Patterson et al., 1997; Tewari et al., 1999), teniendo un papel deter- minante en la estabilidad microbiológica de este producto.

En las muestras de paté no tratadas (control), y tras el almacenamiento en refrigeración durante 30 días, se observa un aumento en el recuento de mohos y levaduras del orden de 4,5 unidades logarítmicas. En consecuencia el producto no reúne las condiciones higiénico-sanitarias adecuadas para su consumo, haciéndose necesario la aplicación de un tratamiento a fin de proveer de una mayor vida comercial al producto y minimizar los posibles riesgos microbiológicos. Resultados similares encontraron Shwartz et al. (2009) en pasta de aceituna untable almacenada tanto a refrigeración como a temperatura ambiente. La vida útil del producto obtenida por estos investigadores fue de hasta 90 días, resultado elevado si lo comparamos con nuestro tratamiento control almacenado en refrigeración, que a los 30 días superaba el recuento microbiano establecido reglamentariamente. Esto puede ser debido a que en la elaboración del paté en nuestro ensayo la cantidad de sorbato potásico adicionada fue de $0,05 \mathrm{~g} / \mathrm{kg}$, un $98 \%$ inferior a la adicionada por Shwartz et al. (2009), que además añadieron, para prevenir el desarrollo de mohos (Tortora et al., 1993), benzoato de sodio (1 g/kg) y T.B.H.Q. $(0,2 \mathrm{~g} / \mathrm{kg})$. Además, estos investigadores

Tabla 2

Cambios microbiológicos (log UFC/gr) en el paté de aceituna tratado con altas presiones hidrostáticas (APH) y mediante tratamiento térmico a dia 30

\begin{tabular}{|c|c|c|c|c|c|c|c|c|c|c|c|c|}
\hline \multirow[b]{3}{*}{ Día 30} & \multirow{2}{*}{\multicolumn{2}{|c|}{ Control }} & \multirow{2}{*}{\multicolumn{2}{|c|}{$\begin{array}{l}\text { Tratamiento } \\
\text { térmico }\end{array}$}} & \multicolumn{4}{|c|}{ APH (450 MPa) } & \multicolumn{4}{|c|}{ APH (600 MPa) } \\
\hline & & & & & \multicolumn{2}{|c|}{$5 \mathrm{~min}$} & \multicolumn{2}{|c|}{$10 \mathrm{~min}$} & \multicolumn{2}{|c|}{$5 \mathrm{~min}$} & \multicolumn{2}{|c|}{$10 \mathrm{~min}$} \\
\hline & Media & $D E$ & Media & $D E$ & Media & $D E$ & Media & $D E$ & Media & $D E$ & Media & $D E$ \\
\hline Mesófilos & $5,2 \mathrm{c}$ & $\pm 0,1$ & $2,6 a$ & $\pm 0,2$ & $2,7 a$ & $\pm 0,1$ & $2,6 a$ & $\pm 0,3$ & $3,6 b$ & $\pm 0,4$ & $3,1 a b$ & $\pm 0,2$ \\
\hline Psicrotrofos & $5,1 b$ & $\pm 0,1$ & $2,4 a$ & $\pm 0,5$ & $2,4 \mathrm{a}$ & $\pm 0,2$ & $2,4 a$ & $\pm 0,6$ & $2,6 a$ & $\pm 0,0$ & $2,6 a$ & $\pm 0,1$ \\
\hline Enterobacterias & nd & & nd & & nd & & nd & & nd & & nd & \\
\hline E. coli & nd & & nd & & nd & & nd & & nd & & nd & \\
\hline Mohos y levaduras & $5,1 \mathrm{c}$ & $\pm 0,1$ & $2,3 b$ & $\pm 0,5$ & $1,5 a b$ & $\pm 0,7$ & $0,5 a$ & $\pm 0,7$ & nd & & nd & \\
\hline
\end{tabular}

Diferentes letras en la misma fila indican diferencias estadísticamente significativas (Test de Turkey p $<0,05$ ). ns: no significativo. nd: no detectado por debajo del límite de detección. Los resultados se expresan como media y DE (desviación estándar). 
añadieron ácido láctico para ajustar el pH y aumentar así la vida útil del producto.

Tras el periodo de almacenamiento en refrigeración se observa en general un incremento en el recuento de mohos y levaduras en todos los tratamientos excepto en los tratamientos a $600 \mathrm{MPa}$, en los que no se detectaron la presencia de estos microorganismos por debajo del límite de detección del método. El tratamiento control sin tratar mostró significativamente valores mayores al resto de tratamientos. Además las muestras tratadas térmicamente a su vez presentaron valores significativamente superiores a los tratamientos por alta presión a $450 \mathrm{MPa}$ (5 y $10 \mathrm{~min}$ ) y a $600 \mathrm{MPa}(5$ y $10 \mathrm{~min}$ ). Por lo que los tratamientos a APH se perfilan como mejor alternativa al tratamiento térmico en la inhibición del desarrollo de mohos y levaduras.

La no presencia de Escherichia coli ni de Enterobacteriaceae tanto en el control como en los diferentes tratamientos (Tabla 1 y 2 ) indican que las condiciones de elaboración fueron adecuadas sanitariamente, los conservantes utilizados fueron los apropiados y los envases utilizados fueron los suficientemente estériles y herméticos. Todo ello, sumado a los recuentos obtenidos para mesófilos, psicrotrofos, mohos y levaduras tras los tratamientos de APH, indica la aptitud del producto para el consumo según las recomendaciones del CODEX ALIMENTARIUS.

El análisis instrumental de color realizado el día de elaboración del producto (Tabla 3), refleja que los patés sometidos a tratamiento por APH (600MPa, $5 \mathrm{~min}$ ) son significativamente más claros que los sometidos al resto de los tratamientos. La coordenada cromática $a^{*}$ (rojo-verde) sólo presentó variaciones significativas en el tratamiento térmico, suponiendo este un aumento de la componente roja del color. No se han encontrado datos bibliográficos respecto a los efectos sobre dicha coordenada de los tratamientos por APH en patés de aceitunas; sin embargo existen resultados, aunque contradictorios, para otros frutos y vegetales. Krebber et al. (2003) encuentran, en zumos de tomate, un aumento de la coordenada $\mathrm{a}^{*}$ al aplicar tratamiento de APH (700 $\mathrm{MPa}$ ) durante 1 minuto. Dicho aumento se atribuye al golpe de calor, y a los efectos de compactación y homogeneización que supone dicho tratamiento. La coordenada cromática b* (amarillo-azul) y el croma $\mathrm{C}_{\mathrm{ab}}{ }^{*}$ no mostraron diferencias significativas con el tipo de tratamiento aplicado a la pasta de aceituna. La no existencia de cambios significativos en el croma es indicativa de que el paté no ha sufrido la degradación de pigmentos, ni la polimerización de los polifenoles (Segovia-Bravo et al., 2009). Para la caracterización de color además de las coordenadas colorimétricas $\left(L^{*}, a^{*}\right.$ y $\left.b^{*}\right)$ y los parámetros psicofísi$\cos \left(\mathrm{C}_{\mathrm{ab}}{ }^{*}, \mathrm{~h}_{\mathrm{ab}}{ }^{*}\right)$ son utilizadas, frecuentemente, relaciones como: $L^{*} a^{*} / b^{*}$ y $a^{*} / b^{*}$. En nuestro estudio, no se han encontrado diferencias significativas en $\mathrm{a}^{\star} / \mathrm{b}^{*}$. Ambas relaciones (Tabla 3 ) presentaron el valor más alto para el tratamiento control siendo significativo en $L^{*} a^{*} / b^{*}$. Criado et al. (2008) atribuyen la presencia de fenómenos de pardeamiento cuando la relación $\mathrm{a}^{*} / \mathrm{b}^{*}$ toma valores positivos alejados del cero. Rodrigo et al. (2007) al aplicar tratamientos de APH (100MPa$700 \mathrm{MPa}$ ) en fresas y zumo de tomate no encuentran diferencias significativas en la relación $L^{*} a^{*} / b^{*}$, relación considerada como una de las mejores para definir la degradación del color. Por el contrario, el ángulo del tono $h_{a b}{ }^{*}$ presenta valores significativamente menores en las muestras sometidas a tratamiento térmico, como consecuencia del aumento significativo de la coordenada $b^{*}$ para dicho tratamiento, y la relación existente entre $b^{*}$ y $h_{a b}{ }^{*}$.Teniendo en cuenta la pasteurización térmica, Escudero-Gilete et al. (2009) no encontraron diferencias significativas de color (CIELAB) entre las pastas de aceituna sometidas a diferentes condiciones de temperatura de pasteurización. Estos autores indican que pasteurizar durante $15 \mathrm{~min}$ a $85^{\circ} \mathrm{C}$ fue la más apropiada temperatura de pasteurización que no conduce significativamente a cambios visuales discernibles en la pasta de aceituna por el consumidor. Al comparar los resultados con este tratamiento empleando la misma variedad de aceituna, observamos que cuando aplica-

Tabla 3

Color instrumental en el paté de aceituna tratado con altas presiones hidrostáticas (APH) y mediante tratamiento térmico tras el procesado (día 1)

\begin{tabular}{|c|c|c|c|c|c|c|c|c|c|c|c|c|}
\hline \multirow[b]{3}{*}{ Día 1} & \multirow{2}{*}{\multicolumn{2}{|c|}{ Control }} & \multirow{2}{*}{\multicolumn{2}{|c|}{$\begin{array}{l}\text { Tratamiento } \\
\text { térmico }\end{array}$}} & \multicolumn{4}{|c|}{ APH (450 MPa) } & \multicolumn{4}{|c|}{ APH (600 MPa) } \\
\hline & & & & & \multicolumn{2}{|c|}{$5 \mathrm{~min}$} & \multicolumn{2}{|c|}{$10 \mathrm{~min}$} & \multicolumn{2}{|c|}{$5 \mathrm{~min}$} & \multicolumn{2}{|c|}{$10 \mathrm{~min}$} \\
\hline & Media & $D E$ & Media & $D E$ & Media & $D E$ & Media & $D E$ & Media & $D E$ & Media & $D E$ \\
\hline$L^{*}$ & $54,9 a$ & $\pm 0,0$ & $54,7 a$ & $\pm 0,9$ & $56,0 a$ & $\pm 1,1$ & $55,9 a$ & $\pm 0,7$ & $57,7 \mathrm{~b}$ & $\pm 0,6$ & $56,4 a b$ & \pm 0 \\
\hline$a^{*}$ & $4,1 a$ & $\pm 0,0$ & $4,7 b$ & $\pm 0,2$ & $4,1 a$ & $\pm 0,2$ & $4,0 a$ & $\pm 0,1$ & $3,9 a$ & $\pm 0,1$ & $4,2 a$ & $\pm 0,2$ \\
\hline $\mathbf{b}^{*}$ & $35,4 \mathrm{~ns}$ & $\pm 0,0$ & 34,4 & $\pm 1,0$ & 35,0 & $\pm 1,1$ & 35,2 & $\pm 1,1$ & 34,2 & $\pm 0,4$ & 34,7 & $\pm 0,8$ \\
\hline $\mathrm{C}_{\mathrm{ab}}{ }^{*}$ & $35,6 \mathrm{~ns}$ & $\pm 0,0$ & 34,8 & $\pm 0,9$ & 35,3 & $\pm 1,2$ & 35,4 & $\pm 1,1$ & 34,4 & $\pm 0,4$ & 35,0 & $\pm 0,8$ \\
\hline $\mathrm{h}_{\mathrm{ab}}{ }^{*}$ & $83,5 b$ & $\pm 0,0$ & $82,2 a$ & $\pm 0,4$ & $83,4 b$ & $\pm 0,2$ & $83,5 b$ & $\pm 0,1$ & $83,5 b$ & $\pm 0,2$ & $83,0 b$ & $\pm 0,2$ \\
\hline$a^{*} / b^{*}$ & $0,2 \mathrm{~ns}$ & $\pm 0,0$ & 0,1 & $\pm 0,0$ & 0,1 & $\pm 0,0$ & 0,1 & $\pm 0,0$ & 0,1 & $\pm 0,0$ & 0,1 & $\pm 0,0$ \\
\hline$L^{*} a^{*} / b^{*}$ & $11.0 \mathrm{~b}$ & $\pm 0,0$ & $5,5 a$ & $\pm 0,2$ & $5,6 a$ & $\pm 0,2$ & $5,6 a$ & $\pm 0,1$ & $5,8 a$ & $\pm 0,2$ & $5,6 a$ & $\pm 0,2$ \\
\hline
\end{tabular}

Diferentes letras en la misma fila indican diferencias estadísticamente significativas (Test de Tukey p < 0,05). ns: no significativo. Los resultados se expresan como media y DE (desviación estándar). 
Tabla 4

Evaluación sensorial del paté de aceituna tratado con altas presiones hidrostáticas (APH) y mediante tratamiento térmico tras el procesado (día 1)

\begin{tabular}{|c|c|c|c|c|c|c|c|c|c|c|}
\hline \multirow[b]{3}{*}{ Día 1} & \multirow{2}{*}{\multicolumn{2}{|c|}{$\begin{array}{l}\text { Tratamiento } \\
\text { térmico }\end{array}$}} & \multicolumn{4}{|c|}{ APH (450 MPa) } & \multicolumn{4}{|c|}{ APH (600 MPa) } \\
\hline & & & \multicolumn{2}{|c|}{$5 \mathrm{~min}$} & \multicolumn{2}{|c|}{$10 \mathrm{~min}$} & \multicolumn{2}{|c|}{$5 \mathrm{~min}$} & \multicolumn{2}{|c|}{$10 \mathrm{~min}$} \\
\hline & Media & $D E$ & Media & $D E$ & Media & $D E$ & Media & $D E$ & Media & $D E$ \\
\hline Aspecto & $5,7 n s$ & $\pm 2,6$ & 7,3 & $\pm 2,0$ & 7,3 & $\pm 2,2$ & 7,5 & $\pm 1,8$ & 7,3 & $\pm 1,8$ \\
\hline Sabor & $6,1 \mathrm{~ns}$ & $\pm 1,9$ & 7,3 & $\pm 1,5$ & 7,2 & $\pm 1,5$ & 6,8 & $\pm 1,7$ & 7,3 & $\pm 2,3$ \\
\hline Textura & $5,5 \mathrm{~ns}$ & $\pm 2,5$ & 6,7 & $\pm 1,6$ & 6,5 & $\pm 2,0$ & 6,7 & $\pm 1,8$ & 7,2 & $\pm 1,8$ \\
\hline
\end{tabular}

Diferentes letras en la misma fila indican diferencias estadísticamente significativas (Test de Tukey p<0,05). ns: no significativo. Los resultados se expresan como media y DE (desviación estándar).

mos un tratamiento térmico de pasteurización a $80^{\circ} \mathrm{C}$ durante $20 \mathrm{~min}$ se obtienen valores de $L^{*}$ un $5.3 \%$ menos claros a los obtenidos por estos investigadores. Además todas las muestras presentaron valores de $a^{*}$ y $b^{*}$ alrededor de 2 y 27 unidades CIELAB respectivamente, inferiores (Tabla 3 ) a los obtenidos en nuestro tratamiento térmico de pasteurización $\left(a^{*}=4.7 y^{*}=34.4\right)$.

Para evaluar la preferencia de los consumidores en el producto final se realizó el análisis sensorial evaluando los parámetros aspecto, sabor y textura de los patés sometidos a APH y a pasteurización (Tabla 4). El tratamiento control no se evaluó sensorialmente por no conocerse datos microbiológicos en el momento de la cata, previniendo así posibles afecciones sobre la salud de los jueces-catadores, ya que, el objetivo de la investigación ha sido el comparar tratamiento térmico de pasteurización versus APH en el paté de aceitunas, por tanto, no es de interés el análisis sensorial a la muestra control, pues en ningún caso, se podrá comercializar este producto sin tratamiento de pasteurización alguno. Además el paté de aceituna elaborado es un producto que tiene que recibir al menos un tratamiento de pasteurización para su comercialización y poder así llegar al consumidor como así revelan los datos microbiológicos obtenidos en nuestro estudio el día 1 de elaboración (Tabla 1). Al ser éste un producto de consumo poco frecuente y con un nicho de mercado específico, la dispersión de los resultados fue elevada $(p>0,05)$ ya que el test de consumidores lo realizaron personal no habituado al consumo de este tipo de paté de aceituna. Sin embargo, se encuentra una tendencia clara de los resultados ya que los distintos tratamientos experimentales afectan la aceptabilidad y la calidad final del producto. En cuanto al aspecto, los tratamientos sometidos a APH obtuvieron una mayor puntuación, entre un $28-31 \%$ más, que el tratamiento térmico. En todos los tratamientos la aceptabilidad sensorial fue alta (Tabla 4). La puntuación dada por los catadores para la textura fue mayor en los tratamientos de APH que en el tratamiento térmico entre un $18 \%$ a $31 \%$. Al igual que la puntuación del sabor entre un $11 \%$ a $20 \%$. Resultados análogos fueron obtenidos por Kouniaki et al. (2004) y Oey et al. (2008). Dichos investigadores indican que los cambios en la composición química y el análisis sensorial de los alimentos tratados por altas presiones son inferiores a los cambios observados en los sometidos a tratamientos térmicos, con respecto a los alimentos "frescos" o no procesados. Sin embargo no se observaron diferencias entre los distintos tratamientos por APH a distintas presiones y tiempos de tratamientos (Tabla 4).

Para determinar la estabilidad oxidativa del producto elaborado se analizó el índice de peróxidos y la estabilidad rancimat (Tabla 5). Al comparar la estabilidad oxidativa de los patés control respecto a

Tabla 5

Cambios en la estabilidad oxidativa del paté de aceituna: Índice de Peróxidos (meq. $\mathrm{O}_{2} / \mathrm{Kg}$ grasa) y Estabilidad Rancimat (h), tratado con altas presiones hidrostáticas (APH) y mediante tratamiento térmico, tras el procesado (día 1) y tras $\mathbf{3 0}$ días de almacenamiento en refrigeración

\begin{tabular}{|c|c|c|c|c|c|c|c|c|c|c|c|c|}
\hline \multirow[b]{3}{*}{ Día 1} & \multirow{2}{*}{\multicolumn{2}{|c|}{ Control }} & \multirow{2}{*}{\multicolumn{2}{|c|}{$\begin{array}{l}\text { Tratamiento } \\
\text { térmico }\end{array}$}} & \multicolumn{4}{|c|}{ APH (450 MPa) } & \multicolumn{4}{|c|}{ APH (600 MPa) } \\
\hline & & & & & \multicolumn{2}{|c|}{$5 \mathrm{~min}$} & \multicolumn{2}{|c|}{$10 \mathrm{~min}$} & \multicolumn{2}{|c|}{$5 \mathrm{~min}$} & \multicolumn{2}{|c|}{$10 \mathrm{~min}$} \\
\hline & Media & $D E$ & Media & $D E$ & Media & $D E$ & Media & $D E$ & Media & $D E$ & Media & $D E$ \\
\hline I. Peróxidos & $30,6 c$ & $\pm 0,1$ & $26,9 b c$ & $\pm 7,3$ & $22,4 b$ & $\pm 3,0$ & $15,9 a$ & $\pm 0,6$ & $25,9 b c$ & $\pm 4,7$ & $22,8 b$ & $\pm 1,4$ \\
\hline Estabilidad & $62,4 a b$ & $\pm 3,8$ & $64,0 a$ & $\pm 10,9$ & $63,5 a b$ & $\pm 5,0$ & $73,1 b$ & $\pm 2,2$ & $62,6 a b$ & $\pm 5,3$ & $63,4 a b$ & $\pm 9,7$ \\
\hline \multicolumn{13}{|l|}{ Día 30} \\
\hline I. Peróxidos & $64,5 d$ & $\pm 0,2$ & $25,9 a b$ & $\pm 6,4$ & $41,9 c$ & $\pm 1,2$ & $20,9 a$ & $\pm 7,7$ & $45,2 c$ & $\pm 13,0$ & $33,0 b$ & $\pm 7,9$ \\
\hline Estabilidad & $32,9 a$ & $\pm 1,0$ & $62,4 c$ & $\pm 8,0$ & $54,0 \mathrm{~b}$ & $\pm 0,6$ & $67,0 \mathrm{~d}$ & $\pm 5,5$ & $56,5 \mathrm{bc}$ & $\pm 13,0$ & $57,5 b c$ & $\pm 2,4$ \\
\hline
\end{tabular}

Diferentes letras en la misma fila indican diferencias estadísticamente significativas (Test de Tukey p $<0,05)$. ns: no significativo.

Los resultados se expresan como media y DE (desviación estándar). 
los procesados mediante tratamiento térmico o de $\mathrm{APH}$, se obtuvieron diferencias significativas en todos los parámetros analizados a día 1 y tras 30 días de almacenamiento a refrigeración. El índice de peróxidos aumentó significativamente durante el periodo de almacenamiento de las muestras a $4{ }^{\circ} \mathrm{C}$ (Shwartz et al., 2009), siendo mayor a los 30 días en todos los tratamientos experimentales excepto en el tratamiento térmico que mantiene prácticamente constante dicho parámetro. Como consecuencia, la estabilidad rancimat disminuyó tras el periodo de almacenamiento. Los valores de estos parámetros reflejan la mejora de la estabilidad oxidativa final del producto frente a los valores obtenidos para el tratamiento control ya que presentan tanto para el índice de peróxidos como para la estabilidad rancimat un aumento y disminución del $50 \%$ aproximado respectivamente.

El índice de peróxidos presentó valores significativamente inferiores en los patés de aceituna procesados a $450 \mathrm{MPa} / 10 \mathrm{~min}$ tanto tras el procesado (15,9 meq $\mathrm{O}_{2} / \mathrm{kg}$ grasa), como tras 30 días de almacenamiento a refrigeración (20,9 meq $\mathrm{O}_{2} / \mathrm{kg}$ grasa) (Tabla 5) no superando en ningún caso el valor de $30 \mathrm{meq}_{2} / \mathrm{kg}$ grasa establecido como límite que indica el estado inicial del deterioro de grasas y aceites por otros investigadores (Gotoh y Wada, 2006). En concordancia con estos resultados, el tratamiento a $450 \mathrm{MPa} / 10 \mathrm{~min}$ fue el que mostró mayor estabilidad rancimat $(73,1 \mathrm{~h}$ tras el procesado y $67,0 \mathrm{~h}$ tras 30 días de almacenamiento a refrigeración). Esto implica una mayor estabilidad oxidativa de los patés así tratados con respecto a los sometidos al resto de tratamientos experimentales. Los antioxidantes como sustratos de las reacciones de oxidación retrasan la de los lípidos (Frankel, 1980). Entre los antioxidantes presentes en la pasta de aceituna se encuentran los tocoferoles, carotenos y compuestos fenólicos (Schwartz et al., 2011) que logran disminuir la oxidación a lo largo del tiempo.

La aplicación de APH presentaría ciertas ventajas frente a los tratamientos térmicos de conservación de alimentos: la inactivación de microorganismos fue similar en ambos tratamientos sin embargo, mediante el tratamiento de alta presión se mejoraría el color y la estabilidad oxidativa del paté tras el almacenamiento. Al comparar el efecto de los 4 tratamientos de alta presión ensayados, el tratamiento a $450 \mathrm{MPa}$ durante $10 \mathrm{~min}$ sería más adecuado que el de mayor presión (600MPa) independientemente del tiempo de aplicación del tratamiento. El aumento de la presión puede producir un aumento de la oxidación de los lípidos insaturados del alimento. Este aumento de la oxidación está relacionado con la desnaturalización de las proteínas causada por la presión, provocando la liberación de iones metálicos que catalizarían la oxidación lipídica (Beltrán et al., 2003). En nuestro ensayo, el índice de peróxidos a 450MPa 10min el día 1 fue aproximadamente un 35\% inferior a los tratamientos con mayor presión. A presiones elevadas, la actividad de la enzima polifenoloxidasa en cebolla mostró un aumento del $30 \%$ después de un tratamiento a 600MPa durante 10min a temperatura ambiente (Buzt et al., 1994). Además, Keenan et al. (2011) demuestran que muestras tratadas por APH a $450 \mathrm{MPa}$ tienen mayores niveles de antioxidantes totales, fenoles y contenido de antocianinas que las muestras tratadas a $600 \mathrm{MPa}$. Éstos compuestos juegan un papel primordial para mejorar la estabilidad oxidativa del producto elaborado (Mcinerney et al., 2007; Oey et al., 2008).

La estabilidad oxidativa mejora en el tratamiento a APH 450MPa, $10 \mathrm{~min}$ versus $5 \mathrm{~min}$, y a los tiempos de almacanamiento analizados (1 y 30 días) en un $13 \%$ y $20 \%$ respectivamente, como consecuencia de la reducción en el índice de peróxidos de un $30 \%$ y $50 \%$, respectivamente (Tabla 5 ). Resultados análogos han sido obtenidos por GarcíaPalazón et al. (2004) al aplicar en fresas y frambuesas rojas tratamientos por APH (400MPa) durante 5 y 10 minutos. El tratamiento (400MPa, 10 minutos) proporcionó muestras con mayor estabilidad oxidativa, atribuyendo este resultado a menores porcentajes de activación de enzimas oxidasas, fundamentalmente peroxidasa y polifenoloxidasa.

El tratamiento con APH a 450MPa supone frente al tratamiento a $600 \mathrm{MPa}$ no sólo una mejora en la vida útil del producto alimentario, sino también un ahorro de tiempo y de energía. Sin embargo, habría que valorar si compensa económicamente aplicar tratamientos de $\mathrm{APH}$ de 10min puesto que se reducen el número de ciclos/día que se pueden aplicar con el equipo de APH y por tanto se procesa menos producto que con tratamientos más cortos.

\section{CONCLUSIONES}

El estudio de la vida útil del paté de aceituna elaborado y conservado en refrigeración, indicaría la viabilidad de la aplicación de la tecnología de APH para obtener alimentos con vida útil similar a la obtenida con el tratamiento tradicional de pasteurización, ya que la reducción microbiana del producto fue suficiente como para asegurar una vida comercial adecuada, y dotarle de mejor calidad higiénico-sanitaria; además de proporcionarle una mayor estabilidad oxidativa manteniendo e incluso resaltando sus características cromáticas, como sucede con la luminosidad. Sin embargo, habría que hacer un estudio económico para comprobar si las ventajas que esta novedosa tecnología aporta, son suficientes como para amortizar la inversión exigida para su aplicación.

\section{AGRADECIMIENTOS}

Este estudio ha sido financiado por el proyecto "Obtención de Alimentos mínimamente procesados y Saludables (proyecto Expediente: 1904542, Ministerio de Ciencia e Innovación" (Gobierno de Es- 
paña). N. Franco agradece al FSE y a la Junta de Extremadura la beca concedida (TEC09072). R. Ramírez agradece a FEDER y a INIA el contrato concedido (BOE 10.01.07). Igualmente, desean agradecer a M. García Serrano, J. Hernández Carretero, J.M. García Ballesteros y J. Barahona Nogales su valiosa colaboración en el desarrollo de este trabajo.

\section{REFERENCIAS}

Barbosa-Cánovas GV, Pothakamury UR, Palou E, Swanson BG. 1998. Tecnologías emergentes en la conservación de alimentos, in Barbosa-Cánovas GV, Pothakamury UR, Palou E, Swanson BG (Ed.) Conservación no térmica de alimentos. Marcel Dekker, Inc., New York, 270.

Beltrán E, Pla R, Yuste J, Mor-Mur M. 2003. Lipid oxidation of pressurized and cooked chicken: Role of sodium chloride and mechanical processing on TBARS and hexanal values. Meat Sci. 64, 19-25.

Buzt P, Koller WD, Tauscher B, Wolf S. 1994. Ultra high pressure processing of onions: Chemical and sensory changes. Lebensm. Wiss. Technol. 27, 463-467.

Capellas M, Mor-Mur M, Sendra E, Pla R, Guamis B. 1996. Population of aerobic mesophils and inoculated E.Coli during storage of fresh goat'smilk cheese treated with highpressure. J. Food Protect. 59, 582-587.

Carlez A. 1994. Traitements para hautes pressions d'aliments d'origine musculaire: Destruction microbienne, modificationde couleur, gélification protéique. Thèse, Université Montpelier II. Montpelier.

Cheftel JC, Cullioli J. 1997. Effect of high pressure on meat: a review. Meat Sci. 3, 211-236.

Cheftel JC. 1992. Effect of high hydrostatic pressure on food constituents: An overview, in Balny C, Hayashi R, Heremans K, Masson P (Ed.) High Pressure of Biotechnology. John Libbey Eurotext, Montrouge, 195-209.

CIE. 2004. Colorimetry: technical report CIE15.2, 3rd edn. Vienna, Austria.

Criado MN, Romero MP, Casanovas M, Motilva MJ. 2008. Pigment profile and colour of monovarietal virgen olive oils from Arbequina cultivar obtained during two consecutive crop seasons. Food Chem. 110, 873-880.

De Lamo S, Capellas M, López T, Hernández M, Guamis B, Roig A. 2004. Behavour of Yersinia enterocolitica strains inoculated in model cheese treated with high hydrostatic pressure. J. Food Protect. 68, 528-533.

Escudero-Gilete ML, Meléndez-Martínez AJ, Heredia FJ, Vicario IM. 2009. Optimization of olive-fruit production using a methodological proposal based on a sensory and objective color analysis. Grasas Aceites 60, 396-404.

Frankel EN. 1980. Lipid oxidation. Prog. Lipid Res. 19, 1-22.

García-Palazón A, Suthanthangjai W, Kajda P, Zabetakis I. 2004. The effects of high hydrostatic pressure on $\beta$-glucosidase, peroxidase and polyphenoloxidase in red raspberry (Rubus idaeus) and strawberry (Fragariaxananassa). Food Chem. 88, 7-10.

Gotoh N, Wada S. 2006. The importance of peroxide value in assessing food quality and food safety. J. Am. Oil Chem. Soc. 83, 473-474.

ISO 4833. 1991. Microbiologie. Directives générales pour le dénombrement des micro-or-ganismes. Méthode par comptage des colonies obtenues à $30^{\circ} \mathrm{C}$. International Organitation for Standardization, Genève, Switzerland.
ISO 7402. 1993: Microbiologie. Directives générales pour le dénombrement sans revivification des Enterobacteriaceae. Techinque NPP el méthode par comptage des colonies. International Organitation for Standardization, Genève, Switzerland.

ISO 7954. 1988. Microbiologie. Directives générales pour le dénombrement des levures et moisissures. Technique par comptage des colonies obtenues à $25^{\circ} \mathrm{C}$. International Organitation for Standardization, Genève, Switzerland.

ISO 8586-2. 2008. Guía general para la selección, entrenamiento y control de evaluadores. Parte 2: Evaluadores sensoriales expertos. International Organitation for Standardization, Genève, Switzerland.

ISO 8589. 2007. Análisis sensorial. Guía general para el diseño de salas de catas. International Organitation for Standardization, Genève, Switzerland.

Keenan DF, Rößle C, Gormley R, Butler F, Brunton NP. 2011. Effect of high hydrostatic pressure and thermal processing on the nutritional quality and enzyme activity of fruit smoothies. LWT - Food Sci. Technol., In Press, Corrected Proof, Available online 14 July 2011.

Kouniaki S, Kajda P, Zabetakis I. 2004. The effect of high hydrostatic pressure on anthocyanins and ascorbic acid in blackcurrants (Ribes nigrum). Flavour Fragance. J. 19, 281-286.

Krebbers B, Matser AM, Hoogerwerf SW, Moezelaar R, Tomassen MMM, Van der Berg EW. 2003. Combined high-pressure and thermal treatments for processing of tomato puree: Evaluation of microbial inactivation and quality parameters. Innovative Food Sci. Emerg. Technol. 4, 377-385.

Mcinerney JK, Cathryn A, Seccafien C, Stewart M, Bird AR. 2007. Effects of high pressure processing on antioxidant activity, and total carotenoid content and availability, in vegetables. Innovative. Food Sci. Emerg. Technol. 8, 543-548.

Mor-Mur M. 2010. Alimentos tratados por alta presión. Aspectos nutricionales. Actividad Dietética 14, 53-58.

Oey I, Lille M, Van Loey A, Hendrick M. 2008. Effect of high pressure processing on colour, texture and flavor of fruits and vegetables bases food products. Trends Food Sci. Technol. 19, 320-328.

Oey I, Van Der Planchen I, Van Loey A, Hendrick M. 2007. Does high pressure processing influence nutritional aspects of plant based food systems?. Trends Food Sci. Technol. 19, 300-308.

Pascual-Anderson R, Calderón-Pascual V. 2000. Microbiología alimentaria. Metodología analítica para alimentos y bebidas. Díaz de Santos, S.A, $2^{2}$ edición, Madrid (España), 117-119.

Patterson MF, Margey DM, Mills G, Simpson R, Glimour A. 1997. The effect of high pressure treatment on microorganisms in foods. High Pressure Research, in the Bioscincesand Biothecnology, K. Heremans, (Ed.) Leuven University Press. Leuven, Belgium, 269-272.

Ponce E, Pla R, Sendra E, Guamis B, Mor-Mur M. 1998. Combined effect of nisin and high hydrostatic pressure in inactivation of Listeria innocua and Escherichia coli in liquid wholeegg. Int. J. Food Microbiol. 43, 15-19.

Porreta S, Birzi A, Chizzon C, Vicini E. 1995. Effects of ultra high hydrostatic pressure treatments on the quality of tomato juice. Food Chem. 52, 35-41.

Préstamo G, y Fontecha J. 2008. High pressure treatment on the tofu fatty acids and acylglycerols content. Innov. Food Sci. Emerg. Technol. 8, 188-191. 
Rastogi NK, Rraghavarao KSMS, Nirajan K. 2005. Developments in osmotic dehydration, en Sun DW (Ed.) Emerging technologies for food processing. Elservier, London, 221-249.

Reglero G, Señorans FJ, Ibáñez E. 2005. Supercritical fluid extraction: an alternative to isolating natural food preservatives, en Barbosa-Cánovas GV, Tapia MS, Cano MP. (Ed.) Novel food procesing technologies. CRC Press, New York, 539-553.

Rodrigo D, Van Loey A, Hendrickx M. 2007. Combined termal and high pressure colour degradation of tomato puree and strawberry juice. J. Food Eng. 79, 553-560.

Schwartz M, Quitral V, Daccarett C, Callejas J. 2009. Desarrollo de pasta untable de aceituna variedad Sevillana. Grasas Aceites 60, 451-457.

Schwartz M, Quitral V, Daccarett C, Callejas J. 2011. Efecto de la adición de ajo en la estabilidad y calidad sensorial de una pasta de aceituna. Grasas Aceites 62, 337-343.

Segovia-Bravo K, Jaén-Galán M, García-García P, Garrido-Fernández A. 2009. Browning reactions in olives:
Mechanism and polyphenols envolved. Food Chem. 114, 1380-1352.

Soliva-Fortuny R, Balasa A, Knorr D, Martín-Belloso O. 2009. Effects of pulsed electric fields on bioactive compounds in foods: a review. Trends Food Sci. Technol. 20, 544-556.

Tewari G, Jayas DS, Holley RA. 1999. High pressure processing of foods: an overview. Sci. Aliment. 19, 619-661.

Torres JA, Velazquez G. 2008. Hydrostatic pressure processing of foods, in Jun S, Irudayaraj J. (Ed.) Food Processing Operations Modeling: Design and Analysis. (Second ed.), FL: CRC Press Inc, Boca Ratón, 173-212.

Tortora G, Berdell R, Case C. 1993. Introducción a la microbiología. Acribia S.A (Ed.) Zaragoza, España.

Voight D, Chevalier F, Qian M, y Kelly A. 2010. Effect of high-pressure treatment on microbiology, proteolysis, lipolysis and levels of flavour compounds in mature blue-beined cheese. Innov. Food Sci. Emerg.Technol. 11, 68-77.

Recibido: $11 / 7 / 11$ Aceptado: 19/9/11 